\section{Drs. Tayer-Shifman and Touma reply}

\section{To the Editor:}

We thank Ya-Chi Li, et al ${ }^{1}$ for their interest in our work and their comment on our paper titled "Performance of Screening Tests for Cognitive Impairment in Systemic Lupus Erythematosus" ${ }^{2}$. We agree that the 2 patients presented by Li, et al illustrate the unmet need for early screening, diagnosis, and monitoring of cognitive function status over time in patients with systemic lupus erythematosus (SLE), potentially enabling early intervention/therapy to prevent the accrual of longterm damage and disability, and to provide information to patients to guide compensatory efforts.

As shown in our recently published systematic review and metaanalysis ${ }^{3}$, cognitive impairment (CI) is highly prevalent in patients with SLE, with pooled prevalence of 38\% (95\% CI: 33-43\%). In patients with neuropsychiatric SLE (NPSLE), the pooled prevalence of CI was 39\% (95\% CI: 24-55\%). Thus, screening of patients with SLE for CI should not be restricted to patients with NPSLE. Also, CI is more common in patients with SLE compared to healthy controls (relative risk $2.8,95 \%$ CI: $2.06-3.81$ ) and compared to patients with rheumatoid arthritis (relative risk $1.8,95 \% \mathrm{CI}$ : $1.30-2.49)$.

The diagnosis of CI in patients with SLE is commonly delayed, and we agree on the need for a reliable and valid cognitive screening test that is both time- and cost-effective and easily administered in an ambulatory setting. However, the preferred test for screening those patients is still to be determined. Several tests have been studied including but not limited to the Montreal Cognitive Assessment ${ }^{3}$.

The 2 patients presented had antiphospholipid antibodies [aPL; including anticardiolipin antibodies (aCL), anti- $\beta_{2}$-glycoprotein I (anti- $\beta_{2}$-GPI), and lupus anticoagulant (LAC)]. However, the importance of aPL in the pathogenesis of CI in patients with SLE is not clear, and though some studies report an association of CI with positive aPL status, others do not. In our initial study, no statistical difference in aPL status was found between patients with and without $\mathrm{CI}^{2}$.

The etiology of CI in SLE is multifactorial (inflammatory vs ischemic) and may be related to autoantibody production (e.g., LAC, aCL, anti- $\beta_{2}$ GPI, antiribosomal P, anti-NR2 glutamate receptor), cytokine-mediated inflammation, and microangiopathy. Although the patients presented by Li, et $a l^{1}$ showed positive aPL and nonspecific brain imaging findings, we should bear in mind that CI in patients with SLE often occurs regardless of NPSLE, positive antibodies, or findings on imaging studies. Thus it should not be restricted to a specific clinical, laboratory, or imaging phenotype, but it should be generalized and available to all patients with SLE. The assessment for CI in SLE is complex and challenging and it is necessary to identify the best screening, diagnostic, and evaluative metrics for CI in SLE.

OSHRAT E. TAYER-SHIFMAN, MD, Clinical Research Fellow of Rheumatology; ZAHI TOUMA, MD, PhD, FACP, FACR, University of Toronto Lupus Clinic, Toronto Western Hospital, Centre for Prognosis Studies in the Rheumatic Diseases, Toronto, Ontario, Canada. Address correspondence to Dr. Z. Touma, Centre for Prognosis Studies in the Rheumatic Diseases, Room 1-415 East Wing, Toronto Western Hospital, 399 Bathurst St., Toronto, Ontario M5T 2S8, Canada.E-mail: zahi.touma@uhn.ca

\section{REFERENCES}

1. Li YC, Chang DM, Lu CC. Montreal Cognitive Assessment and magnetic resonance imaging identify cognitive impairment in young patients with neuropsychiatric systemic lupus erythematosus. J Rheumatol 2019;46:653.

2. Nantes SG, Su J, Dhaliwal A, Colosimo K, Touma Z. Performance of screening tests for cognitive impairment in systemic lupus erythematosus. J Rheumatol 2017;44:1583-9.

3. Rayes HA, Tani C, Kwan A, Marzouk S, Colosimo K, Medina-Rosas J, et al. What is the prevalence of cognitive impairment in lupus and which instruments are used to measure it? A systematic review and meta-analysis. Semin Arthritis Rheum 2018;48:240-55.

J Rheumatol 2019;46:6; doi:10.3899/jrheum.181290 